\title{
Design of Fixed Wideband Beamformer through Improved Maximum Energy Approach
}

\author{
Rui Du, Yangyu Fan, and Jianshu Wang \\ School of Electronics and Information, Northwestern Polytechnical University, Xian, Shaanxi 710072, China \\ Correspondence should be addressed to Rui Du; rdu@mail.nwpu.edu.cn
}

Received 9 March 2016; Revised 10 May 2016; Accepted 26 May 2016

Academic Editor: George Tsoulos

Copyright (C) 2016 Rui Du et al. This is an open access article distributed under the Creative Commons Attribution License, which permits unrestricted use, distribution, and reproduction in any medium, provided the original work is properly cited.

\begin{abstract}
A maximum energy approach is investigated in this paper to design fixed wideband beamformer. This approach has been improved by integrating response variation (RV) into the target function to maintain the frequency invariant property of wideband beamformer over the whole passband. Two methods for designing null to suppress interference signal also have been proposed to make the wideband beamformer robust in complicated environment. Comparisons among other methods are provided to illustrate the effectiveness and enhancement of performance of the new approaches.
\end{abstract}

\section{Introduction}

The term beamforming is derived from early spatial filters that were designed for beams in order to receive a signal radiated from a specific location and attenuate others from other locations $[1,2]$. Beamforming is widely adopted by signal processing of radar, sonar, communication, seismology, geophysics, and so on [3-7].

Wideband beamforming has attracted increasing attentions in recent years because of the advantages of wideband signal such as larger channel capacity. Wideband beamforming can be achieved mainly through two approaches: adaptive wideband beamforming and fixed wideband beamformer. Fixed wideband beamformer directly constrains the response of beamformer, and the beam pattern is constant once set. Three approaches are mainly adopted to design the fixed wideband beamformer: iterative optimization, least square approach, and eigenfilter approach [2]. Eigenfilter approach is efficient because no matrix inversion (least square approach) or iteration (iterative optimization) is required during the optimization.

Eigenfilter is a filter whose coefficients are the elements of an eigenvector. It was first proposed to design digital filters and then extended to the design of beamformer [8]. In [9], fixed wideband beamformer was achieved by maximizing the ratio of mainlobe's energy to sidelobe's energy through eigenfilter approach. It can also be achieved by minimizing total least squares error between reference response and responses over wide passband, both in near field and in far field $[9,10]$.

All the methods above have not discussed the design of null for interference, which is of great importance for beamformer to get a good result in complicated environment with inferences. The width of mainlobe varies over the passband. It will produce distortion if the signal is not coming from the main direction. Response variation (RV) was introduced in [11-14] to maintain a good frequency invariant property in wideband beamforming.

An improved maximum energy approach is proposed to design fixed wideband beamformer. In this approach, $\mathrm{RV}$ is integrated into the target function with a trade-off coefficient, which makes it possible to get a good frequency invariant property and constrain the beamformer's response directly at reference frequency simultaneously. Two methods for null designing are discussed: linear constraint approach and null's expanded energy approach. Both methods are transformed into a standard maximum energy problem and can be solved through a generalized vector approach.

The outline of the paper is as follows.

In Section 1, a brief introduction about the wideband beamforming is made. 
In the next section, a TDL (Tapped Delay Line) structure and beam patterns are described. The standard maximum energy problem and generalized vector approach are also discussed in this section.

In Section 3, we will describe the method we proposed with improved target function and simplified constraint. Two methods for designing null are shown as well, both of which can be casted into a standard maximum energy problem.

The methods are tested in Section 4. The results demonstrate the effectiveness of the proposed methods compared with other methods.

\section{Wideband Beamforming through Maximum Energy Approach}

2.1. Wideband Beamforming Model with TDL Structure. A TDL structure is shown in Figure 1, where there is a linear array that consists of $M$ sensors with a $J$ order FIR connecting to each of them.

The symbol " $\triangle$ " in the structure represents a TDL unit which produces a delay of $\tau$, which has been set to sampling time $T_{s}$ for simplicity.

The output of this TDL structure can be expressed as follows:

$$
y(t)=\mathbf{w}^{H} \mathbf{x}(t)
$$

where

$$
\begin{aligned}
& \mathbf{w}=\left[w_{0,0}, \ldots, w_{M-1, J-1}\right]^{T} \\
& \mathbf{x}(t)=\left[x_{0}(t), \ldots, x_{M-1}(t), x_{0}\left(t-T_{s}\right), \ldots,\right. \\
& \left.\quad x_{M-1}\left(t-(J-1) T_{s}\right)\right]^{T},
\end{aligned}
$$

where $\mathbf{w}$ is $M J \times 1$ weight vector and $\mathbf{x}(t)$ is the received signal through TDL structure.

We denote the received signal at the first sensor by $x_{0}(t)$. It can be represented as an inverse Fourier transformation within the bandwidth $\left[\omega_{l}, \omega_{h}\right]$ :

$$
x_{0}(t)=\frac{1}{2 \pi} \int_{\omega_{l}}^{\omega_{h}} X_{0}(\omega) e^{j \omega t} d \omega .
$$

Thus, the signal at $m$ th and $i$ th unit of the TDL structure can be represented as

$$
x_{m, i}(t)=\frac{1}{2 \pi} \int_{\omega_{l}}^{\omega_{h}} X_{0}(\omega) e^{j \omega\left(t-\left(\tau_{m}+i T_{s}\right)\right)} d \omega .
$$

Hence, (1) can be derived in a new form:

$$
\begin{aligned}
y(t) & =\frac{1}{2 \pi} \int_{\omega_{l}}^{\omega_{h}} X_{0}(\omega) e^{j \omega t} \sum_{m=0}^{M-1} \sum_{i=0}^{J-1} e^{-j \omega\left(\tau_{m}+i T_{s}\right)} w_{m, i}^{*} d \omega \\
& =\frac{1}{2 \pi} \int_{\omega_{l}}^{\omega_{h}} X_{0}(\omega) e^{j \omega t} P(\theta, \omega) d \omega,
\end{aligned}
$$

where $P(\theta, \omega)$ is the response of the beamformer and is expressed as

$$
P(\omega, \theta)=\sum_{m=0}^{M-1} \sum_{i=0}^{J-1} e^{-j \omega\left(\tau_{m}+i T_{s}\right)} w_{m, i}^{*}=\mathbf{w}^{H} \mathbf{a}(\omega, \theta),
$$

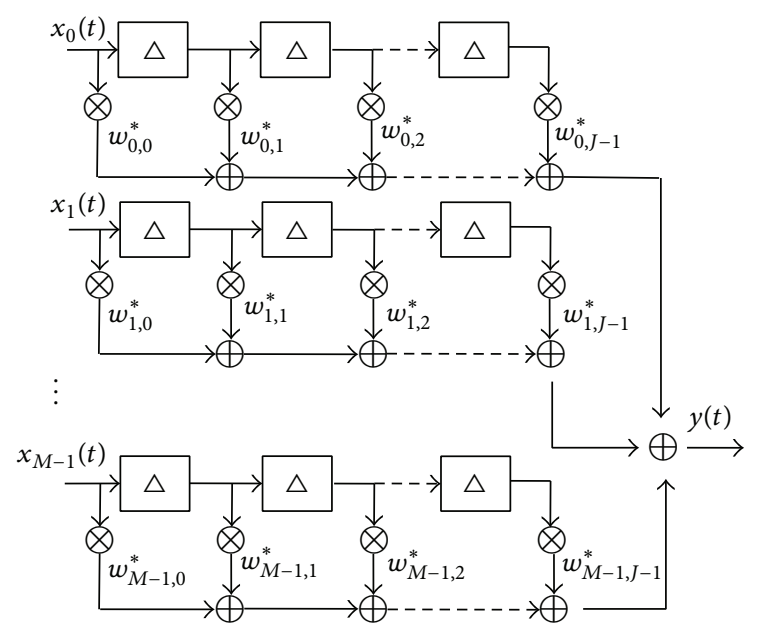

FIgURE 1: Structure of TDL.

where $\mathbf{a}(\omega, \theta)$ is the steering vector of the TDL structure; that is,

$$
\begin{aligned}
& \mathbf{a}(\omega, \theta)=\left[e^{-j \omega \tau_{0}}, \ldots, e^{-j \omega \tau_{M-1}}, e^{-j \omega\left(\tau_{0}+T_{s}\right)}, \ldots,\right. \\
& \left.e^{-j \omega\left(\tau_{M-1}+(J-1) T_{s}\right)}\right]^{T},
\end{aligned}
$$

where $\omega$ is the frequency and $\theta$ is the arriving direction of the signal. $\tau_{0}, \tau_{1}, \ldots, \tau_{M-1}$ is the time delay in the array, which can be computed through $\tau_{m}=m d \times \sin (\theta) / c$, where $m=$ $0, \ldots, M-1$ and $c$ is the speed of light.

For fixed wideband beamformer, $P(\omega, \theta)$ is only related to the weight vector and steering vector of the array.

2.2. Maximum Energy Approach. Maximum energy approach can provide a closed-form solution to the fixed wideband beamformer and it is computationally efficient because no matrix inversion is involved.

Given a Hermitian matrix $\mathbf{R}$ and a positive definite matrix B, generalized Rayleigh ratio is described as

$$
\frac{\mathbf{w}^{H} \mathbf{R w}}{\mathbf{w}^{H} \mathbf{B w}} \text {. }
$$

Equation (8) reaches its maximum when $\mathbf{w}$ is the generalized eigenvector corresponding to the maximum generalized eigenvalue of matrix pair $\mathbf{R}$ and $\mathbf{B}, \mathbf{R w}=\lambda_{\max } \mathbf{B w}$, and reaches its minimum when $\mathbf{w}$ is the generalized eigenvector corresponding to the minimum generalized eigenvalue of matrix pair $\mathbf{R}$ and $\mathbf{B}, \mathbf{R} \mathbf{w}=\lambda_{\min } \mathbf{B w}$.

According to the property of (8), the design of fixed wideband beamformer can be implemented by maximizing the Rayleigh ratio of beamformer's energy on angle range of interest to the energy over the whole direction area on the passband. For simplification, the angle range of interest is referred to as mainlobe in the paper, and the angle range that is not of interest is referred to as sidelobe. Hence,

$$
\max _{\mathbf{w}} \frac{\int_{\Omega_{\mathrm{PB}}} \int_{\Theta_{\mathrm{ML}}}\left|\mathbf{w}^{H} \mathbf{a}(\omega, \theta)\right|^{2} d \theta d \omega}{\int_{\Omega_{\mathrm{PB}}} \int_{\Theta}\left|\mathbf{w}^{H} \mathbf{a}(\omega, \theta)\right|^{2} d \theta d \omega} .
$$


In (9), the numerator is the energy of the beamformer on mainlobe $\Theta_{\mathrm{ML}}$ on passband $\Omega_{\mathrm{PB}}$ and the denominator represents the beamformer's energy over the whole angle region $\Theta$ on $\Omega_{\mathrm{PB}}$.

A variation of (9) is achieved by replacing the beamformer's energy over whole direction area with the energy only on sidelobe:

$$
\max _{\mathbf{w}} \frac{\int_{\Omega_{\mathrm{PB}}} \int_{\Theta_{\mathrm{ML}}}\left|\mathbf{w}^{H} \mathbf{a}(\omega, \theta)\right|^{2} d \theta d \omega}{\int_{\Omega_{\mathrm{PB}}} \int_{\Theta_{\mathrm{SL}}}\left|\mathbf{w}^{H} \mathbf{a}(\omega, \theta)\right|^{2} d \theta d \omega},
$$

which is equivalent to

$$
\max _{\mathbf{w}} \frac{\mathbf{w}^{H} \mathbf{A}_{\mathrm{ML}} \mathbf{w}}{\mathbf{w}^{H} \mathbf{A}_{\mathrm{SL}} \mathbf{w}}
$$

where

$$
\begin{aligned}
\mathbf{A}_{\mathrm{ML}} & =\int_{\Omega_{\mathrm{PB}}} \int_{\Theta_{\mathrm{ML}}} \mathbf{a}(\omega, \theta) \mathbf{a}(\omega, \theta)^{H} d \theta d \omega \\
\mathbf{A}_{\mathrm{SL}} & =\int_{\Omega_{\mathrm{PB}}} \int_{\Theta_{\mathrm{SL}}} \mathbf{a}(\omega, \theta) \mathbf{a}(\omega, \theta)^{H} d \theta d \omega .
\end{aligned}
$$

Maximum energy approach does not guarantee a smooth constant response over different frequencies [2]. Linear constraints $\mathbf{C}^{H} \mathbf{w}=\mathbf{f}$ can be added to get a smooth response at mainlobe by directly constraining the response of the beamformer over different frequencies [2]. C is $N J \times J$ constraint matrix and $\mathbf{f}$ is the $J \times 1$ response vector. Thus, (11) becomes

$$
\begin{aligned}
\max _{\mathbf{w}} & \frac{\mathbf{w}^{H} \mathbf{A}_{\mathrm{ML}} \mathbf{w}}{\mathbf{w}^{H} \mathbf{A}_{\mathrm{SL}} \mathbf{w}} \\
\text { subject to } & \mathbf{C}^{H} \mathbf{w}=\mathbf{f} .
\end{aligned}
$$

Equation (13) can be transformed into another form:

$$
\max _{\widehat{\mathbf{w}}} \frac{\widehat{\mathbf{w}}^{H} \widehat{\mathbf{A}}_{\mathrm{ML}} \widehat{\mathbf{w}}}{\widehat{\mathbf{w}}^{H} \widehat{\mathbf{A}}_{\mathrm{SL}} \widehat{\mathbf{w}}}
$$

$$
\text { subject to } \widehat{\mathbf{C}}^{H} \widehat{\mathbf{w}}=\mathbf{0} \text {, }
$$

where

$$
\begin{aligned}
& \widehat{\mathbf{w}}=\left[\mathbf{w}^{H},-1\right]^{H}, \\
& \widehat{\mathbf{A}}_{\mathrm{ML}}=\left[\begin{array}{cc}
\mathbf{A}_{\mathrm{ML}} & \mathbf{0} \\
\mathbf{0} & 0
\end{array}\right] \text {, } \\
& \widehat{\mathbf{A}}_{\mathrm{SL}}=\left[\begin{array}{cc}
\mathbf{A}_{\mathrm{SL}} & \mathbf{0} \\
\mathbf{0} & 0
\end{array}\right] \text {, } \\
& \widehat{\mathbf{C}}=\left[\begin{array}{ll}
\mathbf{C}^{H} & \mathbf{f}
\end{array}\right]^{H} \text {. }
\end{aligned}
$$

It is shown in the constraint of (14) that $\widehat{\mathbf{w}}$ locates in the null space of $\widehat{\mathbf{C}}$. Equation (14) can be transformed into a standard maximum energy problem by introducing a matrix
$\mathbf{Z}$ whose columns are the bases of the null space of $\widehat{\mathbf{C}}$ and it satisfies $\widehat{\mathbf{w}}=\mathbf{Z} \widetilde{\mathbf{w}}$. Hence,

$$
\max _{\widetilde{\mathbf{w}}} \frac{\widetilde{\mathbf{w}}^{H} \mathbf{Z}^{H} \widehat{\mathbf{A}}_{\mathrm{ML}} \mathbf{Z} \widetilde{\mathbf{w}}}{\widetilde{\mathbf{w}}^{H} \mathbf{Z}^{H} \widehat{\mathbf{A}}_{\mathrm{SL}} \mathbf{Z} \widetilde{\mathbf{w}}} .
$$

Equation (16) is a standard maximum energy problem and can be solved through generalized vector approach. As we can see in the first equation in (15), the first $M J$ elements of $\widehat{\mathbf{w}}$ can be regarded as $\mathbf{w}$ only when the last element of $\widehat{\mathbf{w}}$ has been scaled to -1 .

\section{Improved Maximum Energy Approach}

3.1. Maximum Energy Approach with Response Variation. RV describes the Euclidean distance between responses at reference frequency and responses at other frequencies within whole angle-frequency region of interest.

In [11-14, 16], a general form of RV is defined:

$$
\begin{aligned}
\mathrm{RV} & =\int_{\Omega_{\mathrm{PB}}} \int_{\Theta}\left|\mathbf{w}^{H} \mathbf{a}(\omega, \theta)-\mathbf{w}^{H} \mathbf{a}\left(\omega_{r}, \theta\right)\right|^{2} d \theta d \omega \\
& =\mathbf{w}^{H} \mathbf{A}_{\mathrm{RV}} \mathbf{w},
\end{aligned}
$$

where

$$
\begin{gathered}
\mathbf{A}_{\mathrm{RV}}=\int_{\Omega_{\mathrm{PB}}} \int_{\Theta}\left(\mathbf{a}(\omega, \theta)-\mathbf{a}\left(\omega_{r}, \theta\right)\right) \\
\cdot\left(\mathbf{a}(\omega, \theta)-\mathbf{a}\left(\omega_{r}, \theta\right)\right)^{H} d \theta d \omega,
\end{gathered}
$$

where $\Theta$ represents the range of angles RV measured.

The introduction of $\mathrm{RV}$ factor makes it possible to constrain response directly on the reference frequency. The energy of mainlobe and sidelobe also can be simplified as follows:

$$
\begin{aligned}
\mathbf{w}^{H} \mathbf{a}\left(\omega_{r}, \theta_{d}\right) & =1 \\
\mathbf{A}_{\mathrm{ML} r} & =\int_{\Theta_{\mathrm{ML}}} \mathbf{a}\left(\omega_{r}, \theta\right) \mathbf{a}\left(\omega_{r}, \theta\right)^{H} d \theta \\
\mathbf{A}_{\mathrm{SL} r} & =\int_{\Theta_{\mathrm{SL}}} \mathbf{a}\left(\omega_{r}, \theta\right) \mathbf{a}\left(\omega_{r}, \theta\right)^{H} d \theta .
\end{aligned}
$$

Equation (19) constrains the response of beamformer at looking direction $\theta_{d}$ without distortion.

Equation (13) can be transformed into a new form with $\mathrm{RV}$ and new constraint:

$$
\begin{aligned}
\max _{\mathbf{w}} & \frac{\mathbf{w}^{H} \mathbf{A}_{\mathrm{ML} r} \mathbf{w}}{\mathbf{w}^{H}\left(\mathbf{A}_{\mathrm{SL} r}+\alpha \mathbf{A}_{\mathrm{RV}}\right) \mathbf{w}} \\
\text { subject to } & \mathbf{w}^{H} \mathbf{a}\left(\omega_{r}, \theta_{d}\right)=1,
\end{aligned}
$$

where $\alpha$ is a positive trade-off coefficient, usually larger than 1, affecting the frequency invariant (FI) property. The beamformer will achieve better FI property with larger $\alpha$. However, if the value of $\alpha$ is too large, the whole beam patterns will be degraded. 

(14):

Equation (22) can be transformed into a similar form to

$$
\max _{\mathbf{w}} \frac{\widehat{\mathbf{w}}^{H} \widehat{\mathbf{A}}_{\mathrm{MLr}} \widehat{\mathbf{w}}}{\widehat{\mathbf{w}}^{H} \widehat{\mathbf{A}}_{\mathrm{SR} r} \widehat{\mathbf{w}}}
$$

subject to $\widehat{\mathbf{C}}^{H} \widehat{\mathbf{w}}=0$,

where

$$
\begin{aligned}
\widehat{\mathbf{C}} & =\left[\begin{array}{c}
\mathbf{a}\left(\omega_{r}, \theta_{d}\right) \\
1
\end{array}\right], \\
\widehat{\mathbf{A}}_{\mathrm{ML} r} & =\left[\begin{array}{cc}
\mathbf{A}_{\mathrm{ML} r} & \mathbf{0} \\
\mathbf{0} & 0
\end{array}\right], \\
\widehat{\mathbf{A}}_{\mathrm{SR} r} & =\left[\begin{array}{cc}
\left(\mathbf{A}_{\mathrm{SL} r}+\alpha \mathbf{A}_{\mathrm{RV}}\right) & \mathbf{0} \\
\mathbf{0} & 0
\end{array}\right], \\
\widehat{\mathbf{w}} & =\left[\begin{array}{ll}
\mathbf{w}^{H} & -1
\end{array}\right]^{H} .
\end{aligned}
$$

Similarly, (23) can be transformed into a new standard maximum energy problem:

$$
\max _{\widehat{\mathbf{w}}} \frac{\widetilde{\mathbf{w}}^{H} \mathbf{Z}^{H} \widehat{\mathbf{A}}_{\mathrm{MLr}} \mathbf{Z} \widetilde{\mathbf{w}}}{\widetilde{\mathbf{w}}^{H} \mathbf{Z}^{H} \widehat{\mathbf{A}}_{\mathrm{SR} r} \mathbf{Z} \widetilde{\mathbf{w}}} .
$$

Equation (25) reaches its maximum when $\widetilde{\mathbf{w}}$ is the generalized eigenvector corresponding to the largest generalized eigenvalue of matrix pair $\mathbf{Z}^{H} \widehat{\mathbf{A}}_{\mathrm{ML}} \mathbf{Z}$ and $\mathbf{Z}^{H} \widehat{\mathbf{A}}_{\mathrm{SR}} \mathbf{Z}$.

\subsection{Design of Null Point}

3.2.1. Design of Null with Linear Constraint on Null's Response. Null can be generated by directly imposing a constraint at interference angle $\theta_{i}$ at reference frequency $\omega_{r}$ :

$$
\mathbf{w}^{H} \mathbf{a}\left(\omega_{r}, \theta_{i}\right)=\varepsilon,
$$

where $\varepsilon$ is a small positive value, usually smaller than 1 , of the response at null point. Null's response in decibels can be computed through $-20 \lg \varepsilon \mathrm{dB}$.

With this constraint, (22) is rewritten as

$$
\begin{aligned}
\max _{\mathbf{w}} & \frac{\mathbf{w}^{H} \mathbf{A}_{\mathrm{ML} r} \mathbf{w}}{\mathbf{w}^{H}\left(\mathbf{A}_{\mathrm{SL} r}+\alpha \mathbf{A}_{\mathrm{RV}}\right) \mathbf{w}} \\
\text { subject to } & \mathbf{w}^{H} \mathbf{a}\left(\omega_{r}, \theta_{d}\right)=1 \\
& \mathbf{w}^{H} \mathbf{a}\left(\omega_{r}, \theta_{i}\right)=\varepsilon .
\end{aligned}
$$

Hence, (27) can be transformed into a similar form to (14):

$$
\max _{\mathbf{w}} \frac{\widehat{\mathbf{w}}^{H} \widehat{\mathbf{A}}_{\mathrm{ML} r} \widehat{\mathbf{w}}}{\widehat{\mathbf{w}}^{H} \widehat{\mathbf{A}}_{\mathrm{SR} r} \widehat{\mathbf{w}}}
$$

subject to $\widehat{\mathbf{C}}^{H} \widehat{\mathbf{w}}=0$, where

$$
\begin{aligned}
\widehat{\mathbf{C}} & =\left[\begin{array}{cc}
\mathbf{a}\left(\omega_{r}, \theta_{d}\right) & \mathbf{a}\left(\omega_{r}, \theta_{i}\right) \\
1 & \varepsilon
\end{array}\right], \\
\widehat{\mathbf{A}}_{\mathrm{ML} r} & =\left[\begin{array}{cc}
\mathbf{A}_{\mathrm{ML} r} & \mathbf{0} \\
\mathbf{0} & 0
\end{array}\right], \\
\widehat{\mathbf{A}}_{\mathrm{SR} r} & =\left[\begin{array}{cc}
\left(\mathbf{A}_{\mathrm{SL} r}+\alpha \mathbf{A}_{\mathrm{RV}}\right) & \mathbf{0} \\
\mathbf{0} & 0
\end{array}\right], \\
\widehat{\mathbf{w}} & =\left[\begin{array}{ll}
\mathbf{w}^{H} & -1
\end{array}\right]^{H} .
\end{aligned}
$$

And (28) changes into an unconstrained form by introducing a matrix $\mathbf{Z}$ which satisfies $\widehat{\mathbf{w}}=\mathbf{Z} \widetilde{\mathbf{w}}$ :

$$
\max _{\widetilde{\mathbf{w}}} \frac{\widetilde{\mathbf{w}}^{H} \mathbf{Z}^{H} \widehat{\mathbf{A}}_{\mathrm{MLr}} \mathbf{Z} \widetilde{\mathbf{w}}}{\widetilde{\mathbf{w}}^{H} \mathbf{Z}^{H} \widehat{\mathbf{A}}_{\mathrm{SR} r} \mathbf{Z} \widetilde{\mathbf{w}}} .
$$

Equation (30) is a standard maximum energy problem and reaches its maximum when $\widetilde{\mathbf{w}}$ is the generalized eigenvector corresponding to the largest generalized eigenvalue of matrix pair $\mathbf{Z}^{H} \widehat{\mathbf{A}}_{\mathrm{MLr}} \mathbf{Z}$ and $\mathbf{Z}^{H} \widehat{\mathbf{A}}_{\mathrm{SR} r} \mathbf{Z}$.

3.2.2. Design of Null with Expanded Null's Energy. Null's energy at reference frequency is defined as

$$
\begin{aligned}
\int_{\Theta_{\mathrm{NL}}}\left|\mathbf{w}^{H} \mathbf{a}\left(\omega_{r}, \theta\right)\right|^{2} d \theta & =\mathbf{w}^{H} \mathbf{A}_{\mathrm{NL} r} \mathbf{w} \\
\mathbf{A}_{\mathrm{NL} r} & =\int_{\Theta_{\mathrm{NL}}} \mathbf{a}\left(\omega_{r}, \theta\right) \mathbf{a}\left(\omega_{r}, \theta\right)^{H} d \theta .
\end{aligned}
$$

Null is designed for interference, which usually locates in sidelobe: $\Theta_{\mathrm{NL}} \subset \Theta_{\mathrm{SL}}$.

Then, (22) changes to a new form by replacing $\mathbf{A}_{\mathrm{SL} r}$ with $\mathbf{A}_{\mathrm{SL} r}+\beta \mathbf{A}_{\mathrm{NL} r}$ :

$$
\max _{\mathbf{w}} \frac{\mathbf{w}^{H} \mathbf{A}_{\mathrm{MLr}} \mathbf{w}}{\mathbf{w}^{H}\left(\mathbf{A}_{\mathrm{SL} r}+\beta \mathbf{A}_{\mathrm{NL} r}+\alpha \mathbf{A}_{\mathrm{RV}}\right) \mathbf{w}}
$$

subject to $\mathbf{w}^{H} \mathbf{a}\left(\omega_{r}, \theta_{d}\right)=1$,

where $\beta$ is the expansion coefficient which is also a large positive value used to expand null's energy in target function. Commonly, null is lower with larger $\beta$. The value of $\beta$ is related to the actual beamformer, usually bigger than 1000 for a null low enough.

Thus, (32) can be transformed into a new form like (14):

$$
\max _{\mathbf{w}} \frac{\widehat{\mathbf{w}}^{H} \widehat{\mathbf{A}}_{\mathrm{ML} r} \widehat{\mathbf{w}}}{\widehat{\mathbf{w}}^{H} \widehat{\mathbf{A}}_{\mathrm{SRN} r} \widehat{\mathbf{w}}}
$$

subject to $\widehat{\mathbf{C}}^{H} \widehat{\mathbf{w}}=0$, 
where

$$
\begin{aligned}
\widehat{\mathbf{C}} & =\left[\begin{array}{c}
\mathbf{a}\left(\omega_{r}, \theta_{d}\right) \\
1
\end{array}\right], \\
\widehat{\mathbf{A}}_{\mathrm{ML} r} & =\left[\begin{array}{cc}
\mathbf{A}_{\mathrm{ML} r} & \mathbf{0} \\
\mathbf{0} & 0
\end{array}\right], \\
\widehat{\mathbf{A}}_{\mathrm{SRN} r} & =\left[\begin{array}{cc}
\left(\mathbf{A}_{\mathrm{SL} r}+\beta \mathbf{A}_{\mathrm{NL} r}+\alpha \mathbf{A}_{\mathrm{RV}}\right) & \mathbf{0} \\
\mathbf{0} & 0
\end{array}\right], \\
\widehat{\mathbf{w}} & =\left[\begin{array}{ll}
\mathbf{w}^{H} & -1
\end{array}\right]^{H} .
\end{aligned}
$$

Similarly, (33) can be transformed into a standard maximum energy problem:

$$
\max _{\widehat{\mathbf{w}}} \frac{\widetilde{\mathbf{w}}^{H} \mathbf{Z}^{H} \widehat{\mathbf{A}}_{\mathrm{MLr}} \mathbf{Z} \widetilde{\mathbf{w}}}{\widetilde{\mathbf{w}}^{H} \mathbf{Z}^{H} \widehat{\mathbf{A}}_{\mathrm{SRN} r} \mathbf{Z} \widetilde{\mathbf{w}}} .
$$

Equation (35) reaches its maximum when $\widetilde{\mathbf{w}}$ is the generalized eigenvector corresponding to the largest generalized eigenvalue of matrix pair $\mathbf{Z}^{H} \widehat{\mathbf{A}}_{\mathrm{MLr}} \mathbf{Z}$ and $\mathbf{Z}^{H} \widehat{\mathbf{A}}_{\mathrm{SRN} r} \mathbf{Z}$.

\section{Performance Analysis}

In this section, we will test and analyze the performance of the methods proposed. The relative coefficients will be discussed as well.

4.1. Experiment 1. The simulations are performed based on the specifications as follows:

(i) A TDL structure with $M=14$ and $J=20$ is adopted during the simulation.

(ii) Half of the sampling frequency $f_{s}$ has been scaled to $\pi$ and has been sampled to 64 narrow bands; the wide passband is set as $\Omega_{\mathrm{PB}}=[0.5 \pi, \pi]$, and the reference frequency is set as $\omega_{r}=0.7 \pi$.

(iii) Space between elements is half of the wavelength corresponding to the highest frequency of passband.

(iv) Desired signal is coming from $0^{\circ}$, the interference signal comes from $50^{\circ}$, and null is set to be $\left[48^{\circ}, 52^{\circ}\right]$.

(v) Mainlobe is set as $\Theta_{\mathrm{ML}}=\left[-15^{\circ}, 15^{\circ}\right]$ and sidelobe is set to be $\Omega_{\mathrm{SL}}=\left[-90^{\circ},-20^{\circ}\right] \cup\left[20^{\circ}, 90^{\circ}\right]$, and the whole direction area is sampled every $1^{\circ}$.

(vi) $\alpha=10$ and $\varepsilon=10^{-4}$ in method $1 ; \alpha=200$ and $\beta=$ $9 \times 10^{4}$ in method 2 .

(vii) To get a real-value $\mathbf{w}$, all the matrices during the computation are the real part of the original matrices.

Beamformer's responses through the 2 proposed methods are shown in Figures 2 and 3, respectively.

Good beam patterns have been achieved with distortionless mainlobes point at $0^{\circ}$ and nulls reach $-80 \mathrm{~dB}$ or lower.

To illustrate the effectiveness of the proposed methods described in (27) and (32), we compare them with the method

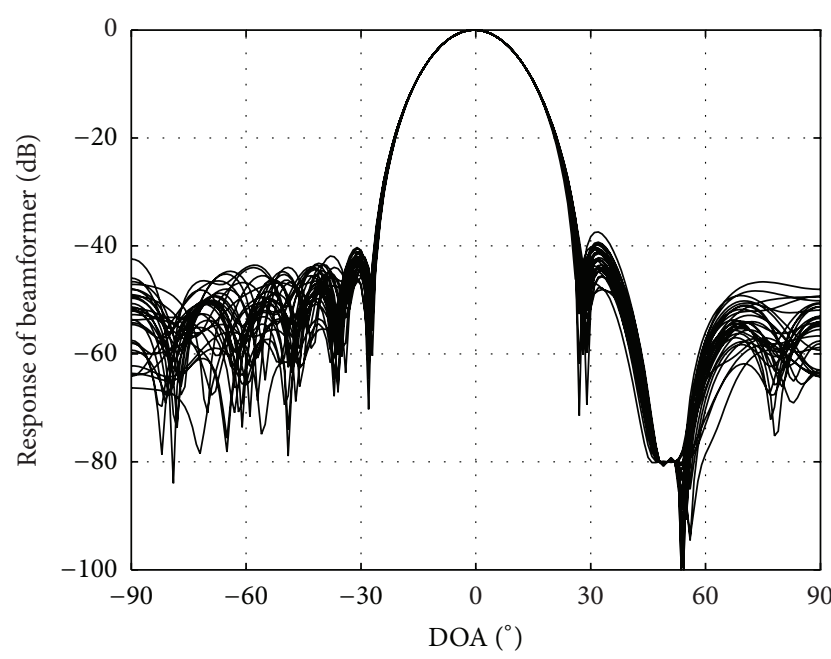

FIGURE 2: Response of beamformer with proposed method 1.

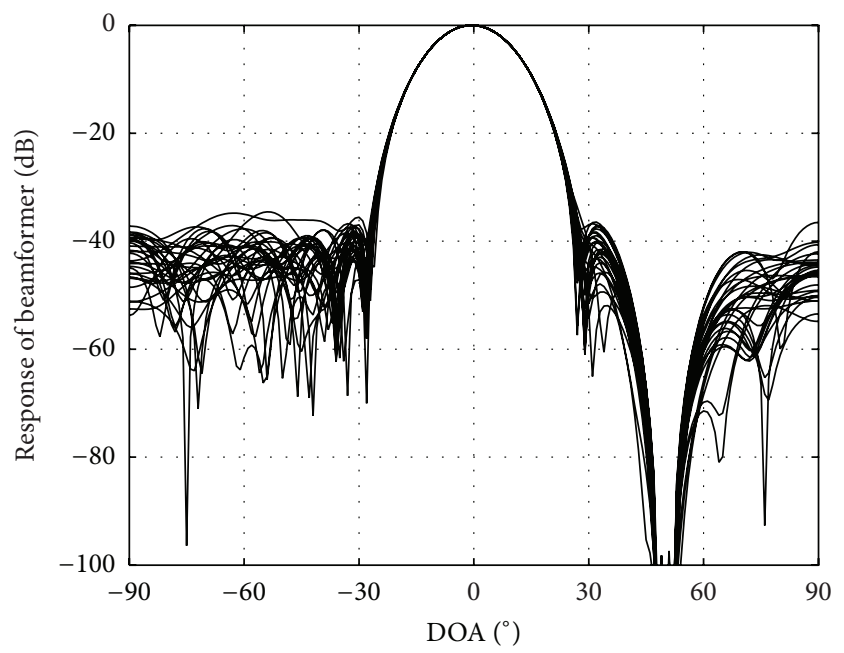

FIGURE 3: Response of beamformer with proposed method 2.

from $[2,15]$. In [15], the design of wideband beamformer was achieved through convex optimization. The weighting function in [15] has been set as $F(\omega, \theta)=1, \delta=1 \times 10^{-6}$, $\varepsilon=10^{-4}$

We test these methods versus width of mainlobe over passband, RV value of mainlobe, peak sidelobe level (SLL) of the beam patterns averaged over passband, and suppression values of null point.

The results are shown in Table 1.

The nulls generated by these four methods are constrained to $-80 \mathrm{~dB}$, which is very good suppression for interference signal. It can be seen in Table 1 with RV taken into consideration during the design of fixed wideband beamformer that both the proposed methods and the method in [15] achieved constant beam width and smaller RV; however, the values through the proposed methods are smaller. The SLL produced by the proposed methods are more than $10 \mathrm{~dB}$ lower than those from the method in $[2,15]$, indicating better suppression over the sidelobe region. 
TABLE 1: Evaluation of four methods.

\begin{tabular}{lcccc}
\hline Measurement & Method in $[2]$ & Method in $[15]$ & Method 1 & Method 2 \\
\hline Width of beam & {$\left[-12^{\circ}, 11^{\circ}\right]$} & {$\left[-10^{\circ}, 9^{\circ}\right]$} & {$\left[-10^{\circ}, 9^{\circ}\right]$} & $1.98 \times 10^{-10}$ \\
RV of mainlobe & $7.69 \times 10^{-3}$ & $3.27 \times 10^{-8}$ & $\left.-10^{\circ}, 9^{\circ}\right]$ & $3.96 \times 10^{-11}$ \\
SLL $(\mathrm{dB})$ & -21.06 & -27.03 & -44.2 & -38.21 \\
\hline
\end{tabular}

TABLE 2: Evaluation of the two proposed methods with different $\alpha$.

\begin{tabular}{lcccc}
\hline \multirow{2}{*}{$\alpha$} & \multicolumn{2}{c}{ Method 1 } & \multicolumn{2}{c}{ Method 2 } \\
& RV & SLL (dB) & RV & SLL (dB) \\
\hline 1 & $6.85 \times 10^{-8}$ & -45.21 & $1.75 \times 10^{-7}$ & -41.71 \\
10 & $1.98 \times 10^{-10}$ & -44.2 & $2.21 \times 10^{-9}$ & -40.99 \\
20 & $1.76 \times 10^{-10}$ & -44.04 & $6.60 \times 10^{-10}$ & -40.23 \\
50 & $1.07 \times 10^{-10}$ & -43.37 & $7.61 \times 10^{-10}$ & -38.51 \\
100 & $6.44 \times 10^{-11}$ & -42.69 & $4.04 \times 10^{-11}$ & -38.39 \\
200 & $4.81 \times 10^{-11}$ & -42 & $3.69 \times 10^{-11}$ & -38.21 \\
500 & $9.61 \times 10^{-12}$ & -40.55 & $3.17 \times 10^{-11}$ & -37.41 \\
1000 & $1.81 \times 10^{-12}$ & -39.17 & $4.19 \times 10^{-11}$ & -37.52 \\
\hline
\end{tabular}

In order to investigate how the RV and SLL change with different $\alpha$, more simulations have been implemented and the results are shown in Table 2 (all the specifications stay the same except for $\alpha$ ).

As we mentioned previously, larger $\alpha$ provide a small RV (better frequency invariant property), but, at the same time, SLL gets higher. One conclusion can be drawn from Table 2: $\mathrm{RV}$ will not keep decreasing with the increasing of $\alpha$ (method 2; RV with $\alpha=1000$ is larger than that with $\alpha=500$ ). A good beam pattern should be a balance between the RV and SLL with an appropriate $\alpha$.

4.2. Experiment 2. Wideband beamformer's performance decreases while the passband gets wider [2]. Wider passband is discussed in this part to test the effectiveness of the proposed methods. The specifications are described as follows:

(i) A TDL structure with $M=12$ and $J=12$ is adopted during the simulation.

(ii) Half of the sampling frequency $f_{s}$ has been scaled to $\pi$ and has been sampled to 64 narrow bands; the wide passband is set as $\Omega_{\mathrm{PB}}=[0.3 \pi, \pi]$, and the reference frequency is set as $\omega_{r}=0.6 \pi$.

(iii) Space between elements is half of the wavelength corresponding to the highest frequency of passband.

(iv) Desired signal is coming from $10^{\circ}$, the interference signal comes from $-40^{\circ}$, and null is set to be $\left[-42^{\circ},-38^{\circ}\right]$.

(v) Mainlobe is set as $\Theta_{\mathrm{ML}}=\left[-5^{\circ}, 25^{\circ}\right]$ and sidelobe is set to be $\Omega_{\mathrm{SL}}=\left[-90^{\circ},-10^{\circ}\right] \cup\left[30^{\circ}, 90^{\circ}\right]$, and the whole direction area is sampled every $1^{\circ}$.

(vi) $\alpha=10$ and $\varepsilon=10^{-3}$ in method $1 ; \alpha=20$ and $\beta=$ $5 \times 10^{3}$ in method 2 .

(vii) To get a real-value $\mathbf{w}$, all the matrices during the computation are the real part of the original matrices.

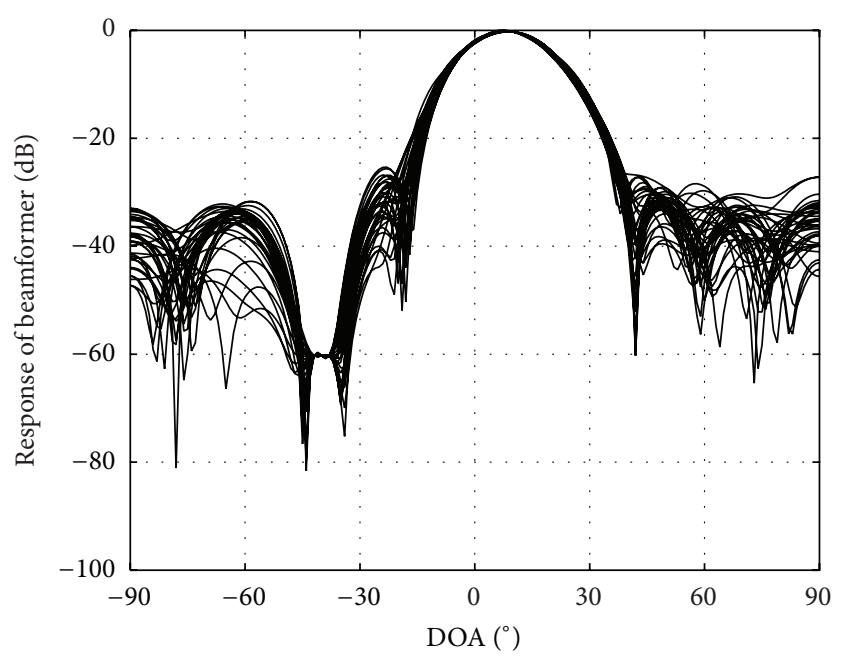

FIGURE 4: Response of beamformer with proposed method 1.

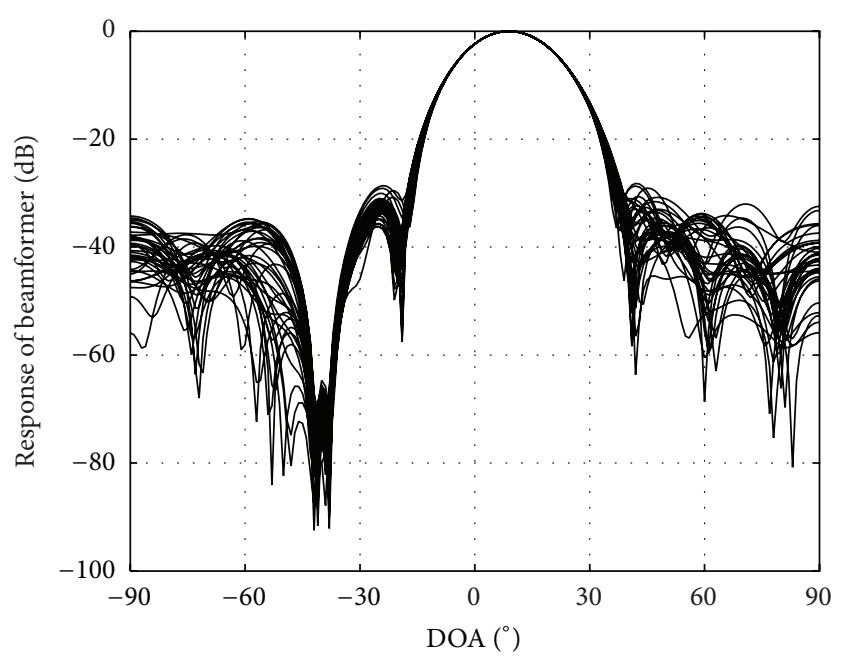

FIGURE 5: Response of beamformer with proposed method 2.

Beamformers' responses through the 2 proposed methods are shown in Figures 4 and 5, respectively.

The beam patterns obtained are shown in Figures 4 and 5 . The mainlobes for both beam patterns are distortionless and point at $10^{\circ}$ as desired. All realized nulls are $-60 \mathrm{~dB}$ or less.

Similarly, the proposed methods are compared with the methods from $[2,15]$. The weighting function in [15] has been set as $F(\omega, \theta)=1, \delta=1 \times 10^{-5}, \varepsilon=10^{-3}$. The results are shown in Table 3.

All the nulls reach $-60 \mathrm{~dB}$. The performances of four methods decrease when we compare Table 3 with Table 1, due 
TABLE 3: Evaluation of four methods.

\begin{tabular}{lcccc}
\hline Measurement & Method in [2] & Method in [15] & Method 1 & Method 2 \\
\hline Width of beam & {$\left[-3^{\circ}, 22^{\circ}\right]$} & {$\left[0^{\circ}, 19^{\circ}\right]$} & {$\left[0^{\circ}, 19^{\circ}\right]$} & $2.31 \times 10^{-8}$ \\
RV of mainlobe & $6.11 \times 10^{-2}$ & $7.74 \times 10^{-7}$ & $\left.-19^{\circ}\right]$ \\
SLL $(\mathrm{dB})$ & -18.21 & -22.64 & -30.24 & $1.07 \times 10^{-10}$ \\
\hline
\end{tabular}

TABLE 4: Evaluation of the two proposed methods with different $\alpha$.

\begin{tabular}{lcccc}
\hline \multirow{2}{*}{$\alpha$} & \multicolumn{2}{c}{ Method 1 } & \multicolumn{2}{c}{ Method 2 } \\
& RV & SLL $(\mathrm{dB})$ & RV & SLL $(\mathrm{dB})$ \\
\hline 1 & $8.31 \times 10^{-8}$ & -32.26 & $2.00 \times 10^{-9}$ & -34.67 \\
10 & $2.31 \times 10^{-8}$ & -30.24 & $7.07 \times 10^{-11}$ & -32.48 \\
20 & $1.64 \times 10^{-8}$ & -29.69 & $1.07 \times 10^{-10}$ & -32.81 \\
50 & $1.42 \times 10^{-8}$ & -29 & $1.53 \times 10^{-10}$ & -32.1 \\
100 & $1.86 \times 10^{-8}$ & -28.37 & $5.90 \times 10^{-12}$ & -31.14 \\
200 & $9.99 \times 10^{-9}$ & -27.96 & $5.29 \times 10^{-11}$ & -31.36 \\
500 & $2.50 \times 10^{-9}$ & -25.84 & $1.32 \times 10^{-12}$ & -29.85 \\
1000 & $1.46 \times 10^{-9}$ & -24.87 & $1.42 \times 10^{-12}$ & -29.92 \\
\hline
\end{tabular}

to the wider passband. But the results through the proposed methods are still better than the rest. The mainlobes remain constant over the whole passband, the SLL are more than $8 \mathrm{~dB}$ lower than other methods, and RV through our methods is smaller.

More simulations have been implemented to investigate $\alpha$ and the results are shown in Table 4 .

Similar to the previous discussion, RV decreases with the increasing of $\alpha$ which also causes an increase in SLL (not strictly satisfied in method 2). A good choice of $\alpha$ should take both RV and SLL into consideration. One conclusion can be drawn from Table 4: for a wider passband, using the second method can achieve better beam patterns (smaller RV and lower SLL).

\section{Conclusion}

In this paper, a fixed wideband beamformer designing method based on maximum energy approach and RV has been proposed and investigated, as well as two methods to design null for interferences. Simulations show that good frequency invariant property in mainlobes and stable nulls for interference signal can be achieved through the proposed methods and the sidelobe suppression with the proposed methods is also lower.

\section{Competing Interests}

The authors declare that they have no competing interests.

\section{References}

[1] B. D. Van Veen and K. M. Buckley, "Beamforming: a versatile approach to spatial filtering," IEEE ASSP Magazine, vol. 5, no. 2, pp. 4-24, 1988.

[2] W. Liu and S. Weiss, Wideband Beamforming-Concepts and Techniques, John Wiley \& Sons, 2010.
[3] M. Ström, M. Viberg, and K. Falk, "Robust transceiver design for wideband MIMO radar utilizing a subarray antenna structure," Signal Processing, vol. 93, no. 12, pp. 3541-3552, 2013.

[4] S. D. Somasundaram, "Wideband robust capon beamforming for passive sonar," IEEE Journal of Oceanic Engineering, vol. 38, no. 2, pp. 308-322, 2013.

[5] Z. G. Feng, K. F. C. Yiu, and S. E. Nordholm, "Placement design of microphone arrays in near-field broadband beamformers," IEEE Transactions on Signal Processing, vol. 60, no. 3, pp. 11951204, 2012.

[6] S. Tanaka, A. Harada, M. Sawahashi, and F. Adachi, "Experiments on coherent adaptive antenna array diversity for wideband DS-CDMA mobile radio," IEEE Journal on Selected Areas in Communications, vol. 18, no. 8, pp. 1495-1504, 2000.

[7] B. S. Huang, Y. L. Huang, P. L. Leu, and S. J. Lee, "Estimation of the rupture velocity and fault length of the 2004 SumatraAndaman earthquake using a dense broadband seismic array in Taiwan," Journal of Asian Earth Sciences, vol. 40, no. 3, pp. 762-769, 2011.

[8] J. Makhoul, "On the eigenvectors of symmetric Toeplitz matrices," IEEE Transactions on Acoustics, Speech, and Signal Processing, vol. 29, no. 4, pp. 868-872, 1981.

[9] D. Korompis, K. Yao, and F. Lorenzelli, "Broadband maximum energy array with user imposed spatial and frequency constraints," in Proceedings of the IEEE International Conference on Acoustics, Speech and Signal Processing (ICASSP '94), vol. 4, pp. 529-532, Adelaide, Australia, April 1994.

[10] S. Doclo and M. Moonen, "Comparison of least-squares and eigenfilter techniques for broadband beamforming," in Proceedings of the 3rd IEEE Benelux Signal Processing Symposium (SPS '02), pp. 73-76, Leuven, Belgium, March 2002.

[11] W. Liu and S. Weiss, "Design of frequency invariant beamformers for broadband arrays," IEEE Transactions on Signal Processing, vol. 56, no. 2, pp. 855-860, 2008.

[12] H. Duan, B. P. Ng, C. M. S. See, and J. Fang, "Applications of the SRV constraint in broadband pattern synthesis," Signal Processing, vol. 88, no. 4, pp. 1035-1045, 2008.

[13] Y. Zhao, W. Liu, and R. J. Langley, "Subband design of fixed wideband beamformers based on the least squares approach," Signal Processing, vol. 91, no. 4, pp. 1060-1065, 2011.

[14] Y. Zhao, W. Liu, and R. J. Langley, "Application of the least squares approach to fixed beamformer design with frequencyinvariant constraints," IET Signal Processing, vol. 5, no. 3, pp. 281-291, 2011.

[15] Z. Fan and G.-L. Liang, "Broadband beamforming with minimum sidelobe and constant beamwidth based on convex optimization," Acta Electronica Sinica, vol. 41, no. 5, pp. 943-948, 2013.

[16] S. Doclo and M. Moonen, "Design of far-field and near-field broadband beamformers using eigenfilters," Signal Processing, vol. 83, no. 12, pp. 2641-2673, 2003. 


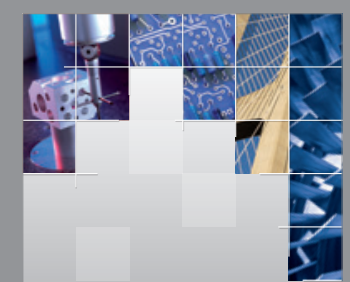

\section{Enfincering}
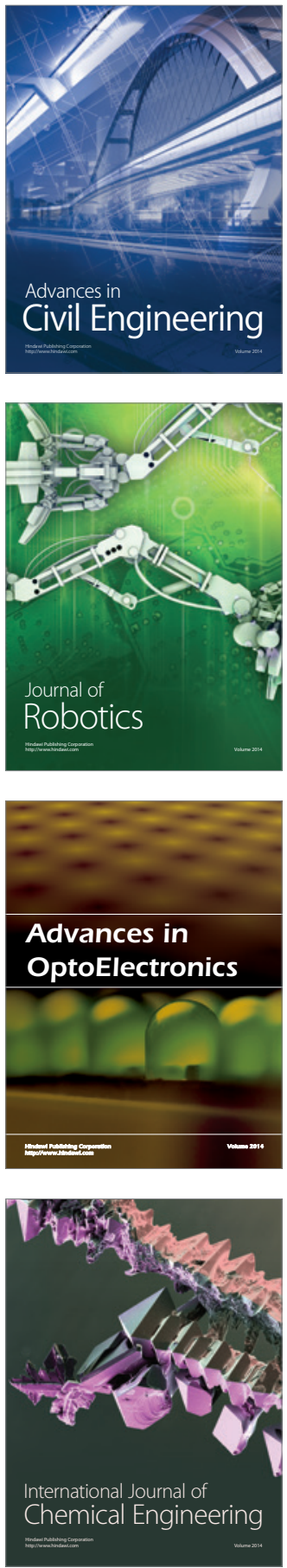

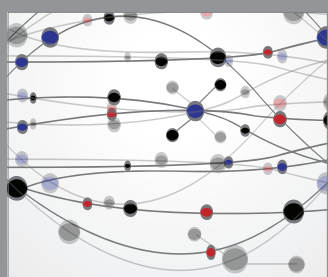

The Scientific World Journal

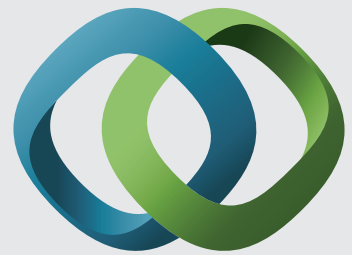

\section{Hindawi}

Submit your manuscripts at

http://www.hindawi.com
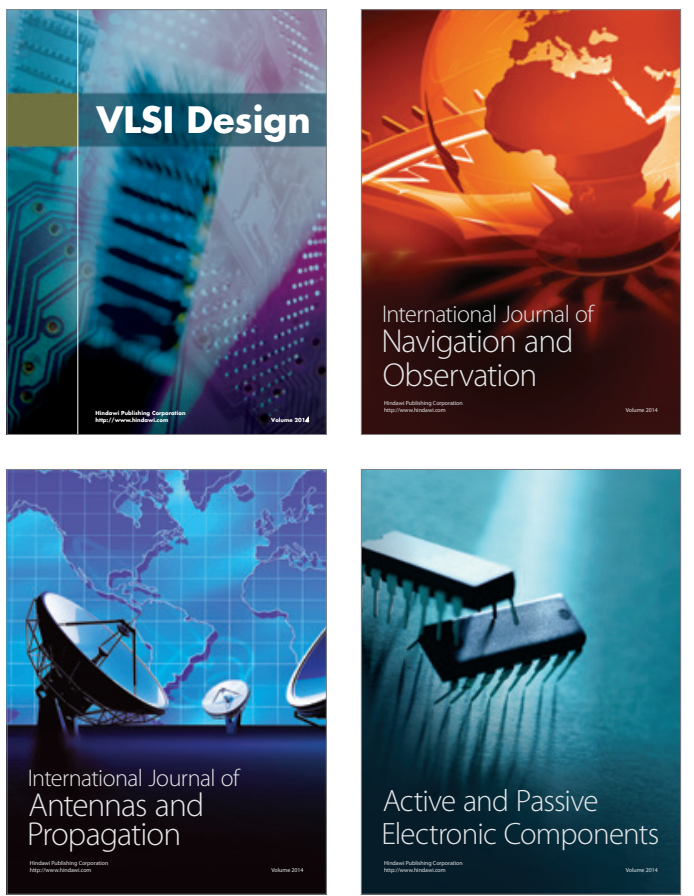
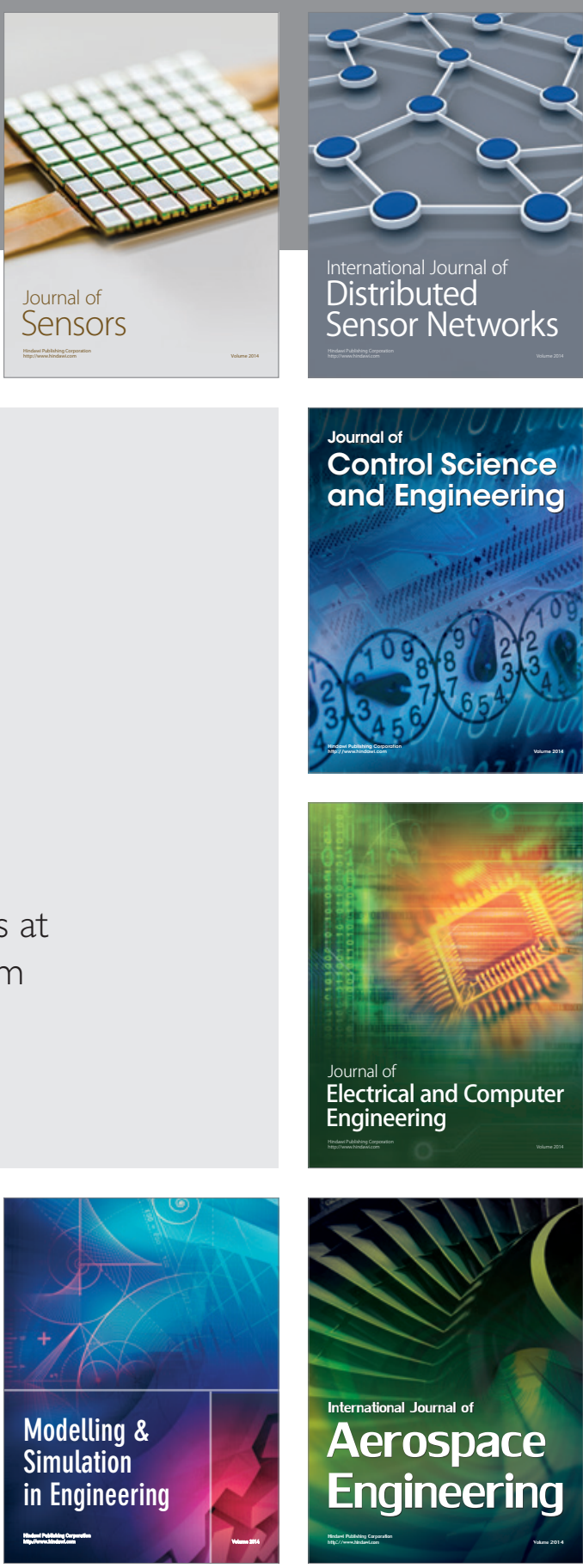

International Journal of

Distributed

Sensor Networks

Journal of

Control Science

and Engineering
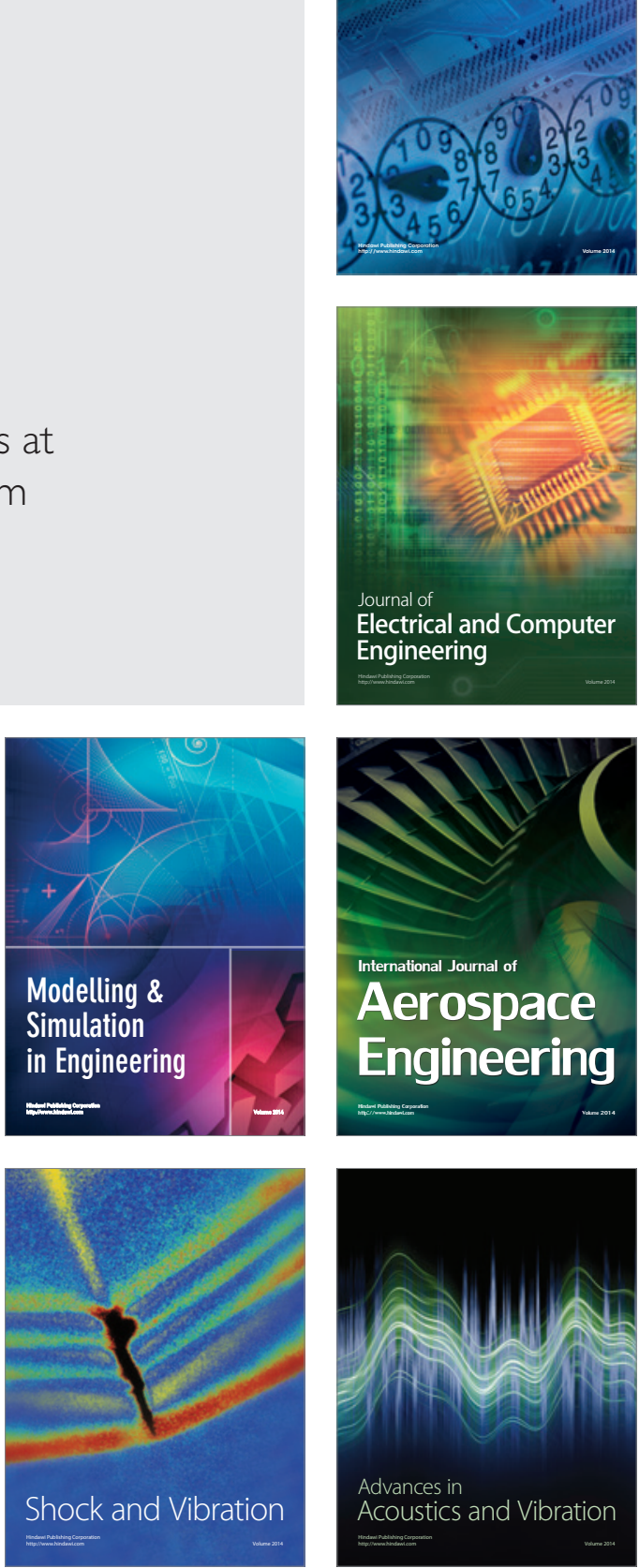